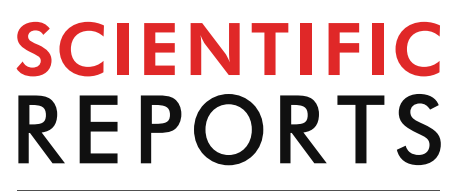

natureresearch

Check for updates

\title{
Adipose-derived exosomal
} miR-210/92a cluster inhibits adipose browning via the FGFR-1 signaling pathway in high-altitude hypoxia

\author{
Yifan Zhang ${ }^{1,2,3,4}$, Kang Song ${ }^{1,2,3,4}$, Gang $\mathrm{Oi}^{4}$, Ranran Yan ${ }^{1}$, Yanqing Yang ${ }^{4}$, Yan Li $^{4}$, \\ Shunjuan Wang ${ }^{1,2,3,4}$, Zhenzhong Bai ${ }^{1,2,3 凶} \&$ Ri-li Ge ${ }^{1,2,3 \boxplus}$
}

Cold and hypoxia are critical drivers of adaptation to high altitudes. Organisms at high altitudes have adapted to maximize the efficiency of oxygen utilization and are less prone to obesity and diabetes than those at low altitudes. Brown adipose tissue (BAT) dissipates energy in the form of heat in both humans and rodents; it also serves to regulate metabolism to curb obesity. However, the role of BAT in high-altitude populations is poorly understood. Serum exosomes can be easily obtained, enabling the study of BAT functions and identification of biomarkers in serum exosomes, both of which contribute to understanding the role of BAT in high-altitude populations. ${ }^{18} \mathrm{~F}$-Fluorodeoxyglucose $\left({ }^{18} \mathrm{~F}\right.$-FDG) positron emission tomography integrated with computed tomography (PET/CT) is the gold standard for studying BAT in human adults. Here, we studied BAT in healthy high-altitude populations via PET/ CT and serum exosomal microRNAs (miRNAs). The observations were validated in mouse tissues and demonstrated that high-altitude hypoxia activated BAT through attenuated white adipose tissue (WAT) secreted exosomal miR-210/92a, which enhanced the FGFR-1 expression in BAT.

Hypoxia exerts profound systemic effects on metabolism. It is implicated in a broad spectrum of metabolic disorders, such as obesity and diabetes ${ }^{1}$. However, the effects of hypobaric hypoxia on adipose tissues is poorly understood. But the mechanisms of high-altitude adaptation have been extensively investigated ${ }^{2-4}$. These mechanisms include enhanced oxygen delivery, efficient consumption of limited oxygen through increased RBC production ${ }^{5,6}$ and reprogramming of both global ${ }^{7}$ and tissue-specific metabolic processes, such as decreases in muscle oxidative phosphorylation and fatty acid oxidation but increases in cellular glycolysis and mitochondrial coupling efficiency ${ }^{8,9}$. These adaptations have enabled populations dwelling long-term at high altitudes to survive. More importantly, these populations have a lower risk of diabetes, obesity, and other metabolic syndromes than sea level populations ${ }^{10-12}$.

Obesity can be caused by excessive accumulation of adipose tissue, which in turn induces hyperlipidemia, cardiovascular diseases, and metabolic syndromes ${ }^{13,14}$. Increased BAT activity is associated with the enhanced heat generation and energy consumption, which in turn confers beneficial effects on adipose development, glucose metabolism and against obesity. Both pharmacological and physiological stimulation can be applied in preclinical approaches to promote BAT activity in human and rodent models. Therefore, revealing the mechanisms modulating BAT development will provide new insights for the prevention and treatment of these metabolic diseases. However, given their limited effects and unclarified side effects, reliable methods to achieve this goal will not be quickly identified. Interestingly, as we have previously reported, native mammals living on the

\footnotetext{
${ }^{1}$ Research Center for High Altitude Medicine, Qinghai University Medical College, Qinghai 810001, Xining, People's Republic of China. ${ }^{2}$ Key Laboratory of High-Altitude Medicine (Qinghai University), Ministry of Education, Qinghai 810001, Xining, People's Republic of China. ${ }^{3}$ Key Laboratory for Application of High-Altitude Medicine in Qinghai Province, Qinghai 810001, Xining, People's Republic of China. ${ }^{4}$ Qinghai Provincial People's Hospital, Qinghai 810007, Xining, People's Republic of China. ${ }^{\circledR}$ email: 2003980001@qhu.edu.cn; geriligao@hotmail.com
} 

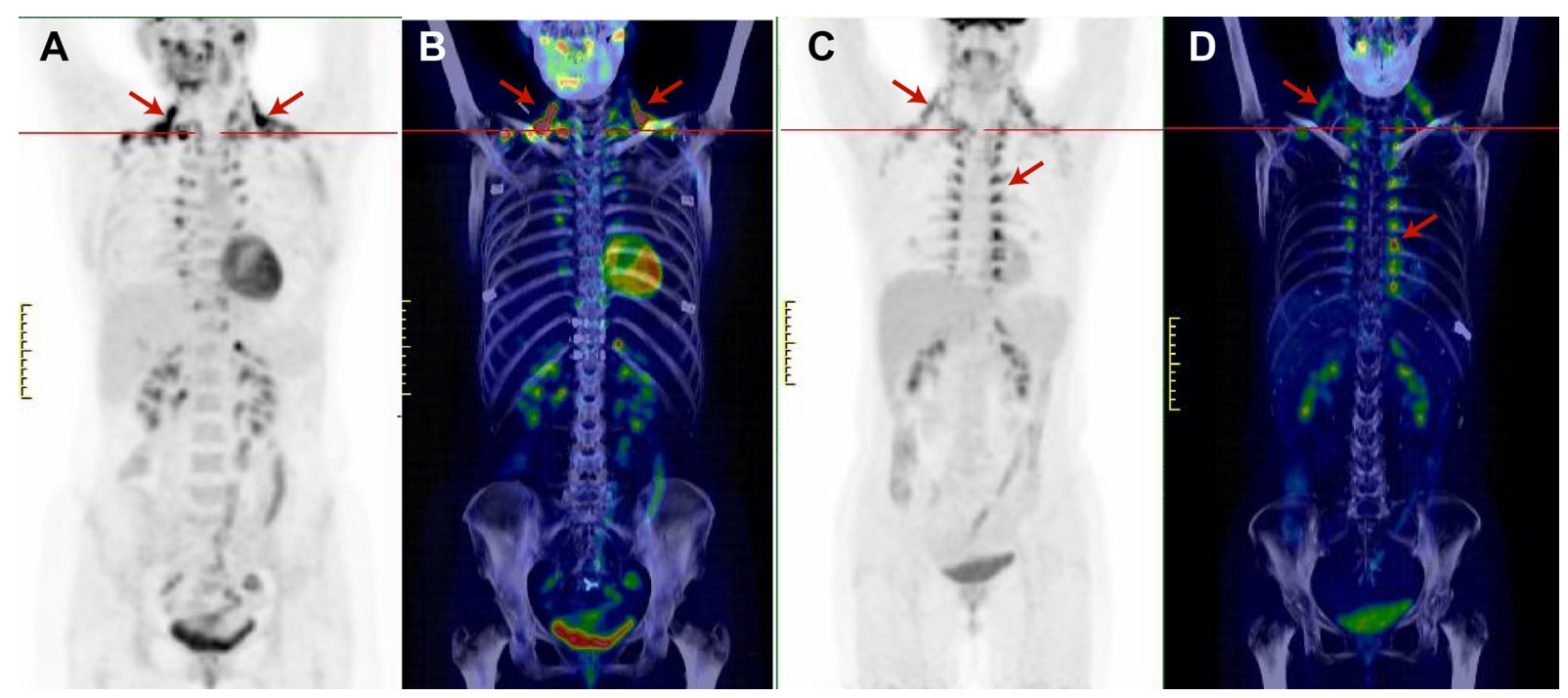

Figure 1. Anterior maximum intensity projection visualization and $3 \mathrm{D}$ mimic images of BAT ${ }^{18} \mathrm{~F}-\mathrm{FDG}$ uptake in the high-BAT group $(\mathbf{A}, \mathbf{B})$ and low-BAT group $(\mathbf{C}, \mathbf{D})$. The arrows indicate the paravertebral-intercostal and mediastinal regions in the neck and supraclavicular/axillary regions.

Qinghai-Tibetan Plateau exhibit increased BAT activity. Therefore, we hypothesize that increased BAT activity could be a new adaptation strategy for high-altitude hypoxia ${ }^{15,16}$.

Exosomes are small circular lipid vesicles with a diameter of $40-100 \mathrm{~nm}$ and unique markers on their membranes. They can be abundantly secreted from adipose tissues and contain many microRNAs (miRNAs) that are released into plasma and taken up by the neighboring or remote recipient cells to modulate multiple biofunctions. Therefore, exosomes have been associated with new cell-cell communication patterns ${ }^{17}$. The involvement of circular miRNAs from exosomes in multiple metabolic modulations, such as insulin resistance and glucose metabolism, along with BAT activation in humans and rodents, has been widely described ${ }^{18}$. More interestingly, exosomal miRNAs play important roles as both endocrines signaling molecules and disease markers. For example, miR-92a is associated with high-density lipoprotein (HDL) components that indicate increased risk of $\mathrm{CVD}^{19}$. However, little is known about BAT activity in high-altitude populations. In this study, we used positron emission tomography integrated with computed tomography (PET/CT) scanning in high-altitude populations to clarify the global BAT amount. In addition, the exosomes were isolated to identify variations in the expression of candidate exosomal miRNAs. Notably, high-altitude-raised mice also showed enhanced BAT activity and similar miRNA alterations in exosomes. Moreover, the exosomal miRNAs originated from WAT and activated BAT through enhanced the FGFR-1, which demonstrated that WAT plays the leading role in the high BAT activity under chronic hypoxia exposure.

\section{Results}

High-altitude hypoxia increased BAT activity in humans and mice. We found 21 scans (21 healthy volunteers) with activated BAT among 239 evaluated ${ }^{18} \mathrm{~F}$-fluorodeoxyglucose $\left({ }^{18} \mathrm{~F}-\mathrm{FDG}\right) \mathrm{PET} / \mathrm{CT}$ scans. In previous reports ${ }^{20}$, five anatomical sites have been used to assess BAT-subphrenic, paravertebral, mediastinal, cervical, and supraclavicular. We selected volunteers evenly distributed by sex, age, BMI, and season. After $6 \mathrm{~h}$ of fasting, all volunteers exhibited various levels of ${ }^{18} \mathrm{~F}-\mathrm{FDG}$ uptake in the above five locations after injection of ${ }^{18} \mathrm{~F}-$ FDG and exposure to $4{ }^{\circ} \mathrm{C}$. We defined the group of individuals with a maximum standard uptake value (SUVmax) greater than 4.5 as the high-BAT group, and the group of individuals with an SUVmax less than 4.5 as the low-BAT group, as shown in Fig. 1. The general characteristics of the subjects are shown in Table 1. The values of BMI, body weight and the other general parameters did not significantly differ between the two cohorts. Interestingly, we found a significant difference in the residence altitude between the two cohorts. Next, we defined the group of individuals residing at an altitude above $3,000 \mathrm{~m}$ as the high-altitude group and the group of individuals residing at an altitude below $3,000 \mathrm{~m}$ as the low-altitude group. The ${ }^{18} \mathrm{~F}-\mathrm{FDG}$ SUVmax of BAT in the two cohorts was significantly different $(p=0.021) 6.831 \pm 1.08$ and $3.872 \pm 0.32$, respectively.

To validate the population study results, C57BL-6J mice were raised at gradient altitudes of 2,300 m and $4,300 \mathrm{~m}$ under the same conditions. We found increases in BAT mitochondrial activity in terms of the oxygen consumption rates (OCRs) in complexes I and II; in addition, the PCR results showed increased levels of browning-selective genes such as $U c p-1, P g c-1 \alpha$ and $P r d m 16$. Furthermore, the Western blot analysis and immunohistochemical staining results showed increased expression of UCP-1 in 4,300 m-raised mice (Fig. 2).

Expression of the miR-210/92a cluster in serum exosomes was negatively correlated with BAT activity. The purity of the exosomes was assessed before miRNA analysis. Initially, the exosomal protein markers CD9 and CD63 were detected by Western blotting. Then, the exosomes were observed with transmis- 


\begin{tabular}{|l|l|l|l|l|}
\hline & Total $(\mathbf{n}=\mathbf{2 1})$ & Low BAT $(\mathbf{n}=\mathbf{1 0})$ & High BAT $(\mathbf{n}=\mathbf{1 1})$ & $\mathbf{p}$ \\
\hline Male/females & $11 / 10$ & $4 / 6$ & $7 / 4$ & - \\
\hline Age $($ years $)$ & $34.67 \pm 2.415$ & $35.10 \pm 2.23$ & $34.27 \pm 2.61$ & 0.45 \\
\hline Height $(\mathrm{m})$ & $1.701 \pm 0.076$ & $1.731 \pm 0.808$ & $1.68 \pm 0.653$ & 0.23 \\
\hline Altitude & $3,111 \pm 862$ & $2,666 \pm 228$ & $3,516 \pm 240$ & $0.02^{*}$ \\
\hline Weight $(\mathrm{kg})$ & $66.57 \pm 8.721$ & $67.90 \pm 9.712$ & $65.36 \pm 7.99$ & 0.85 \\
\hline BMI $\left(\mathrm{kg} / \mathrm{m}^{2}\right)$ & $22.89 \pm 1.599$ & $22.54 \pm 1.73$ & $23.19 \pm 1.47$ & 0.32 \\
\hline Waist/hip & $0.834 \pm 0.099$ & $0.833 \pm 0.115$ & $0.834 \pm 0.89$ & 0.96 \\
\hline Fasting glucose $(\mathrm{mmol} / \mathrm{l})$ & $5.162 \pm 0.511$ & $5.07 \pm 0.54$ & $5.245 \pm 0.49$ & 0.45 \\
\hline RBC $\left(10^{12} / \mathrm{l}\right)$ & $4.729 \pm 0.426$ & $4.780 \pm 0.40$ & $4.682 \pm 0.46$ & 0.61 \\
\hline HGB $(\mathrm{g} / \mathrm{l})$ & $143.9 \pm 15.85$ & $145.9 \pm 14.8$ & $142.1 \pm 17.3$ & 0.59 \\
\hline TCh $(\mathrm{mmol} / \mathrm{l})$ & $4.148 \pm 0.539$ & $3.95 \pm 0.59$ & $4.33 \pm 0.44$ & 0.11 \\
\hline TG $(\mathrm{mmol} / \mathrm{l})$ & $1.754 \pm 0.984$ & $1.53 \pm 1.04$ & $1.96 \pm 0.93$ & 0.34 \\
\hline HDL-C $(\mathrm{mmol} / \mathrm{l})$ & $0.998 \pm 0.151$ & $0.98 \pm 0.12$ & $1.01 \pm 0.18$ & 0.61 \\
\hline LDL-C $(\mathrm{mmol} / \mathrm{l})$ & $2.916 \pm 0.631$ & $2.85 \pm 0.71$ & $2.97 \pm 0.58$ & 0.67 \\
\hline Apo-A1 $(\mathrm{g} / \mathrm{l})$ & $1.300 \pm 0.083$ & $1.27 \pm 0.05$ & $1.33 \pm 0.10$ & 0.12 \\
\hline Apo-B $(\mathrm{g} / \mathrm{l})$ & $1.370 \pm 0.765$ & $1.51 \pm 0.73$ & $1.25 \pm 0.81$ & 0.46 \\
\hline HBA1C $(\%)$ & $5.168 \pm 0.366$ & $5.14 \pm 0.38$ & $5.19 \pm 0.37$ & 0.74 \\
\hline C-peptide $(\mathrm{ng} / \mathrm{ml})$ & $2.532 \pm 0.688$ & $2.43 \pm 0.71$ & $2.63 \pm 0.69$ & 0.52 \\
\hline FINS $(\mu \mathrm{H} / \mathrm{ml})$ & $9.775 \pm 2.196$ & $9.47 \pm 2.20$ & $10.05 \pm 2.26$ & 0.55 \\
\hline miR-210 (FC $)$ & $0.854 \pm 0.393$ & $1.09 \pm 0.41$ & $0.63 \pm 0.21$ & $0.06^{*}$ \\
\hline miR-92a $(\mathrm{FC})$ & $0.747 \pm 0.404$ & $1.08 \pm 0.31$ & $0.45 \pm 0.18$ & $0.00^{*}$ \\
\hline SUV mean & $3.161 \pm 1.799$ & $2.04 \pm 0.56$ & $4.184 \pm 1.94$ & $0.04^{*}$ \\
\hline SUV max & $5.422 \pm 3.031$ & $3.36 \pm 0.67$ & $7.291 \pm 3.14$ & $0.02^{*}$ \\
\hline
\end{tabular}

Table 1. General characteristics of high-altitude adaptation subjects. $B M I$ body mass index, Tch total cholesterol, $T G$ triglyceride, $H B A 1 C$ glycated hemoglobin, $H D L-C$ high density lipoprotein cholesterol, $L D L-C$ low density lipoprotein cholesterol, Apo-A1 Apolipoprotein A1, Apo-B apolipoprotein B, FINS fasting insulin, $S U V$ standard uptake value. ${ }^{\star} F C$ fold change.

sion electron microscopy (TEM), and the exosomal particle sizes and concentration were measured through nanoparticle tracking analysis (NTA). The results are shown in Fig. 3.

Then, to confirm the differential expression of serum exosomal miRNAs between the low-BAT and high-BAT groups, we conducted human MiFinder PCR array testing on eight volunteers. The most abundantly expressed and verified 84 miRNAs in miRbase were probed on the array as candidates, and the expression profiles are shown in the heatmap and the volcano plot (Figs. 4 and 5A). The top significantly differentially expressed miRNAs included miR-92a, miR-30c, miR-99a, miR-210, miR-155, miR-103a, miR-185 and miR-32. Next, we identified various miRNAs from the above results in serum exosomes from all volunteers. Only miR-210 and miR-92a were significantly altered between the high-BAT and low-BAT groups. (Fig. 5B). In addition, a correlation study was performed between the BAT activity indicated by the SUVmax values from PET/CT scanning and the expression levels of the two most frequently identified exosomal miRNA molecules, revealing strong negative associations between them (Fig. 5C-F).

WAT-derived miR-210 and miR-92a synergistically inhibited adipose browning via the FGFR-1 signaling pathway. Next, the effects of high-altitude-derived exosomal miRNA on BAT activity were analyzed. Given the similar features of BAT activity in humans and rodents in previous studies and since human tissues were unavailable, we utilized mice as an alternative. We selected miR-210 and 92a and validated their expression levels in exosomes from various tissues of mice raised at altitudes of 4,300 $\mathrm{m}$ and 2,300 $\mathrm{m}$ for 8 weeks. Interestingly, in the 4,300 m group compared with 2,300 m group, the levels of exosomal miR-210 from serum and WAT were decreased, but those from brain, liver, and lung tissue were significantly increased. The levels of the other tissues did not differ between these groups. In addition, exosomal miR-92a expression was downregulated in serum, WAT and BAT but was unchanged in other tissues (Fig. 6).

To determine the role of miR-210/92a in vivo under high-altitude hypoxia exposure, we used miRNA-210 and -92a agomirs or a mixture of the two to rescue the expression of miR-210 and -92a in WAT of the 4,300 m group via inguinal subcutaneous fat pad injection. We found that the expression of miR-210 and miR-92a in WAT and serum exosomes was efficiently improved by agomir injection. Compared with the 4,300 m- (Ctrl) and negative control (NC) groups, both the miRNA-210 and -92a agomir injection groups exhibited decreased expression of browning-selective genes. Interestingly, the combing group of both miR-210 and miR-92a-agomirs mixture markedly decreased, which have confirmed that miR210/92a cluster have more efficiently suppressed BAT selective gene expression and protein UCP-1 (Figs. 7, 8C).

Based on previous reports ${ }^{21-23}$ together with online programs TargetScanMouse 6.2 (https://www.targetscan .org/) and miRDB (https://www.mirdb.org/), we have identified Fgfr-1, a critical determinant protein of adipose 
A

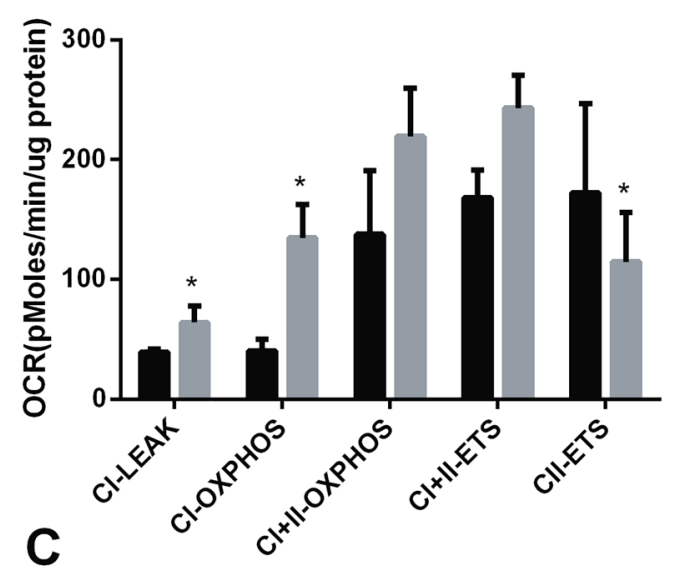

B

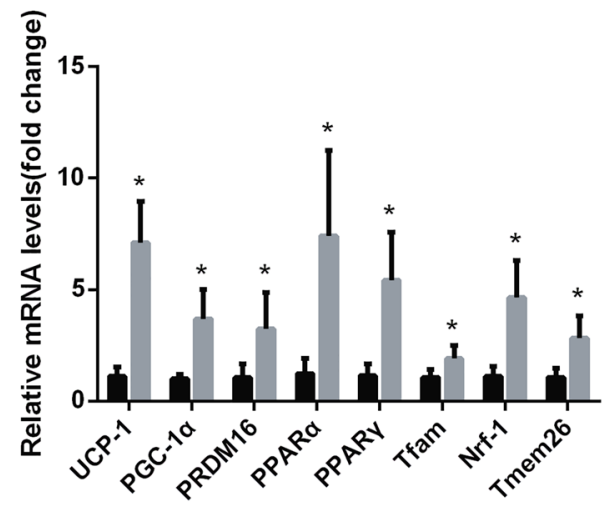

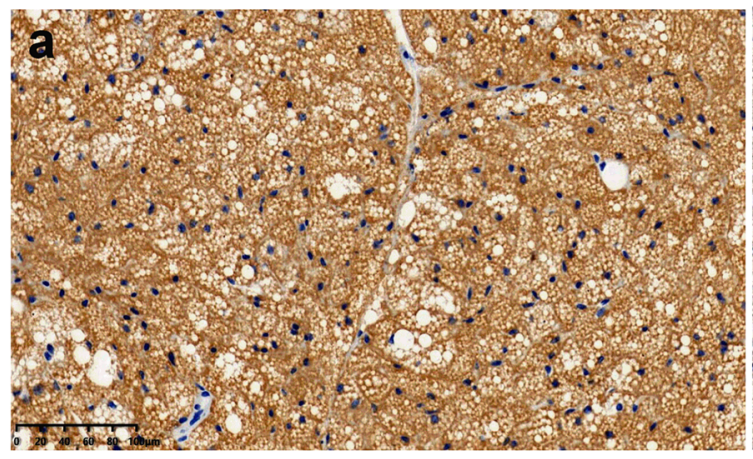

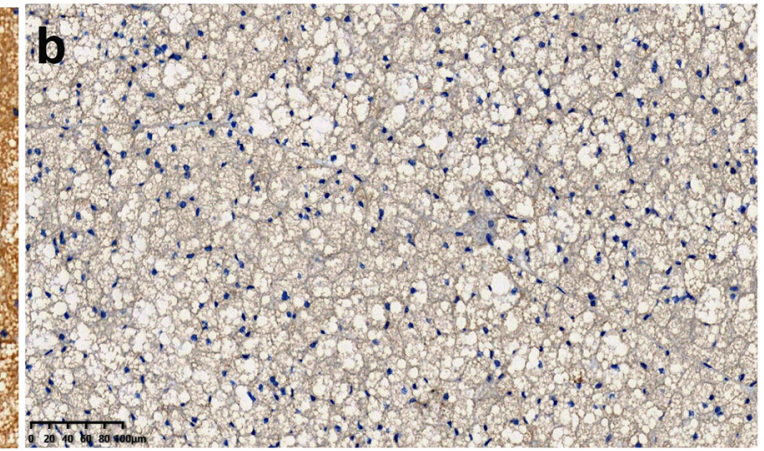

D

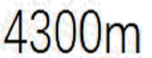

$2300 \mathrm{~m}$

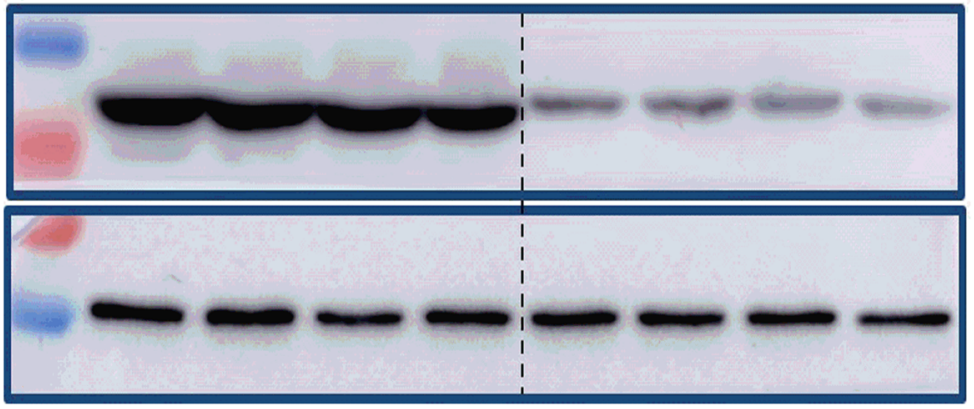

UCP-1(32kDa)

$\alpha-T u b u l i n(55 k D a)$

Figure 2. (A) BAT mitochondrial respiratory capacity in 2,300 $\mathrm{m}$ - and $4,300 \mathrm{~m}$-raised mice ( $\mathrm{n}=5$ per group; $\mathrm{p} \leq 0.05$ was statistically significant). The $2,300 \mathrm{~m}$-raised group was used the control. CI-LEAK is the state 4 respiration rate of respiratory chain complex I. CI-OXPHOS is the mitochondrial respiratory chain complex I state 3 respiration rate, $\mathrm{CI}+\mathrm{II}-\mathrm{OXPHOS}$ is the respiratory chain complexes I+ II state 3 respiration rate, $\mathrm{CI}+\mathrm{II}-\mathrm{ETS}$ is the uncoupling ability, and CII-ETS is the electron transfer capability of respiratory chain complex II. (B) Brown adipose-selective genes' relative expression levels in BAT from mice raised at 2,300 $\mathrm{m}$ or 4,300 $\mathrm{m}$ for 8 weeks. The results were obtained by qPCR analysis. The data were normalized to the levels of $18 \mathrm{~S}$ rRNA in 2,300 m group mice ( $\mathrm{n}=6$ per group; ${ }^{\star} \mathrm{p} \leq 0.05$, Student's $t$ test.) $(\mathbf{C})$ Representative immunohistochemical results in BAT from 2,300 m- and 4,300 m-raised mice [(A) 4,300 m group BAT. (B) 2,300 m group BAT]. Magnification, $\times 200$. (D) Protein expression levels of UCP-1 in BAT from 2,300 m- and 4,300 m-raised mice. 
A
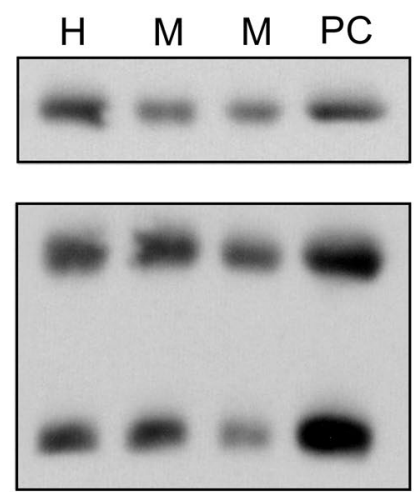

C

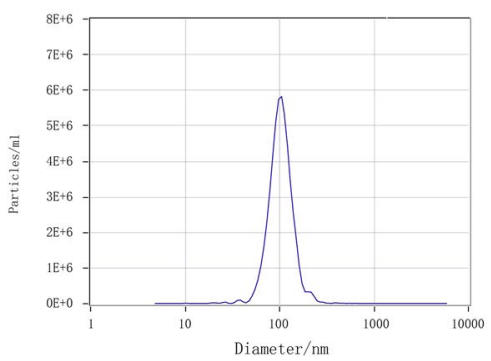

B

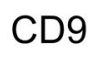

CD63

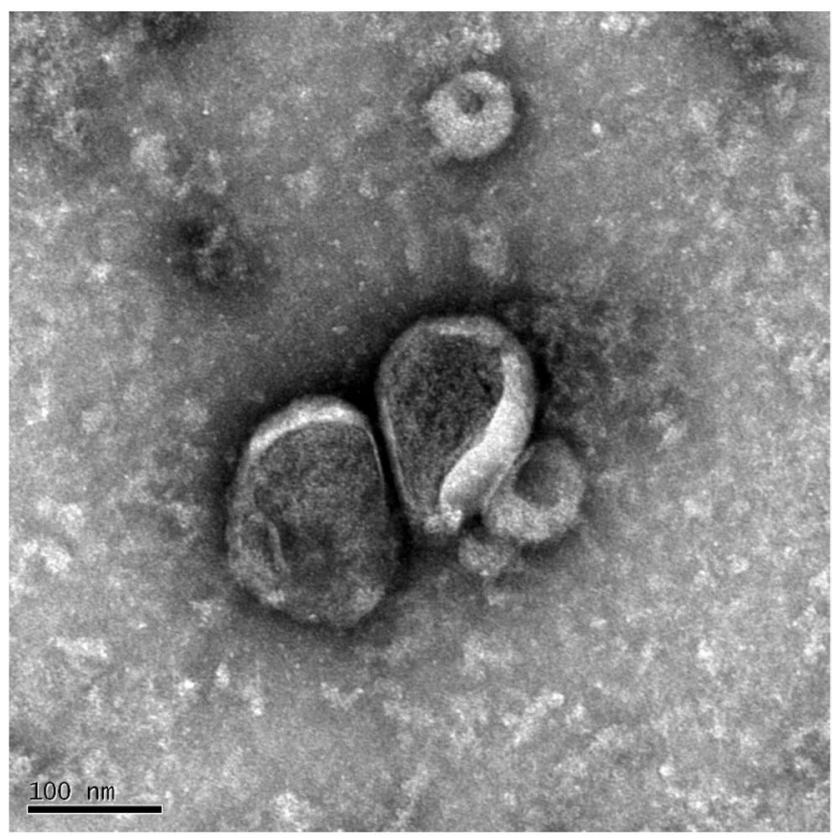

Figure 3. (A) Qualitative Western blot showing the exosomal expression of the exosome marker proteins CD63 and CD9 (PC is for positive control, $\mathrm{M}$ is mouse sample, $\mathrm{H}$ is human sample). (B) Electron micrographs of exosomes in serum (left scale bar, $100 \mathrm{~nm}$ ). (C) The particle size distribution (PSD) of exosomes was measured by nanoparticle tracking analysis; the concentration and zeta potential, by analysis of the particle traces.

browning factors, as a main predicted target of miR-210, even not established in miR-92a. To validate the Fgfr-1 expression altered after high-altitude exposure, we analyzed the expression levels of Fgfr-1 in BAT from 2,300 mand 4,300 m-raised mice and the expression levels both in mRNA and protein of $F g f r-1$ was significantly higher in $4300 \mathrm{~m}$ mice than $2300 \mathrm{~m}$. After injected miR-210-agomir for $4300 \mathrm{~m}$ mice, the Fgfr-1expression was decreased compared with that in the Ctrl $(4,300 \mathrm{~m})$ and NC groups. However, we have not found that any changes in the miR-92a-agomir group. But the expression was the most significantly reduced in miR-210/92a-agomir mixture group, which have compared with injection either miR-210- or miR-92a-agomir alone. (Fig. 8).

HIF-1 $\alpha$ diminished with exposure periods in high-altitude hypoxia. Previous studies indicated that miR-210 is one of the hypoxia-regulated miRNA family members, and have been identified that the augmented parallel expression trends of HIF-1 $\alpha$ (hypoxia-inducible factor $1 \alpha$ ) with miR-210 under hypoxia ${ }^{24}$. To validate the long-term hypoxia whether have influenced on miR-210 and HIF-1a, we have detected the expression of serum exosomal miR-210 and HIF-1 $\alpha$ in WAT at different time points of hypoxic exposure. We found that the expression levels decreased after transit up-forward trends of exosomal miR-210 and WAT HIF-1 $\alpha$ in 4,300 m group mice with the time-dependently. However, the expression levels of exosomal miR-92a decreasing after exposure periods. (Fig. 9).

\section{Discussion}

High-altitude hypoxia reduces body fat accumulation and body weight in both humans and rodents. Several mechanisms, including increased sympathetic nerve tone, appetite loss, modulations in genes encoding enzymes involved in glucose metabolism, and suppression of mitochondrial activity, have been proposed to explain the means by which hypoxia-induced metabolic reprogramming can lead to loss of body mass ${ }^{25}$. Additionally, our previous study reported that exposure to hypoxia combined with cold facilitated phenotypic changes in WAT that enhanced browning ${ }^{15,16}$. However, there is no clear evidence of whether hypoxia, as in the case of highaltitude exposure, can affect BAT activity. This study makes clear associations between hypobaric hypoxia and BAT, indicating that the protective effects of hypoxia might be caused by increased BAT activity. Therefore, this study provides novel ideas to the field.

Given that cold exposure can activate BAT and result in the uptake of a large amount of glucose, we recruited volunteers residing at different altitudes and conducted PET/CT scanning according to SUV data. We found that high-altitude exposure-induced BAT activity was increased significantly in subjects living above an altitude of $3,000 \mathrm{~m}$. Therefore, we hypothesized that high-altitude exposure might be involved in the activation of BAT. To assess whether high-altitude hypoxia could potentially impact adipose tissue, we raised mice at altitudes of $2,300 \mathrm{~m}$ and 4,300 $\mathrm{m}$. As expected, the mice raised at 4,300 m exhibited signs of elevated BAT activation, such as elevated UCP-1 expression in both WAT and BAT, upregulation of BAT-selective genes in WAT, and enhanced 


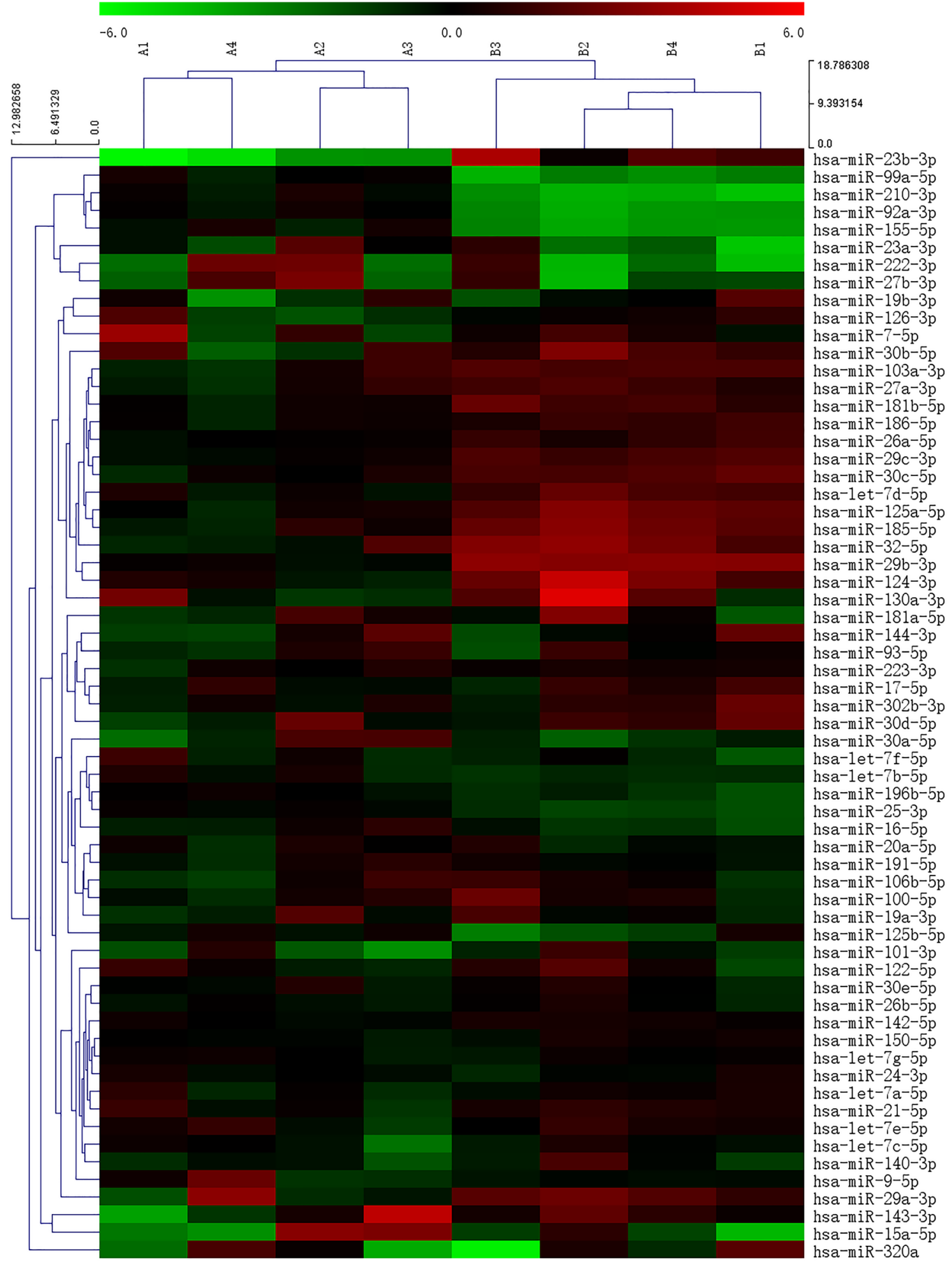

Figure 4. Heatmap of exosomal miRNAs differentially expressed between the high-BAT and low-BAT groups. Hierarchical clustering analysis of the 84 commonly expressed miRNAs by the Euclidean distance method. Each row represents one miRNA, and each column represents one tissue sample. The relative miRNA expression level is depicted according to the color scale. Red indicates elevated expression, and green indicates reduced expression. The numbers 2, 0 , and -2 indicate fold changes along the corresponding spectrum. High-BAT group (B1-4). Paired Low-BAT group (A1-4). 
A

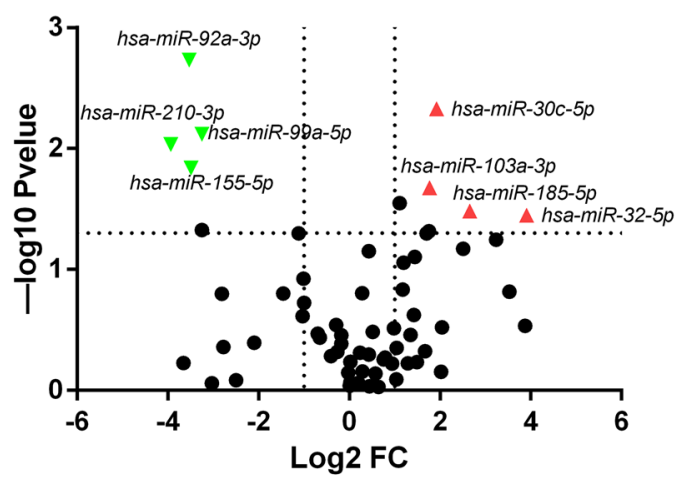

C

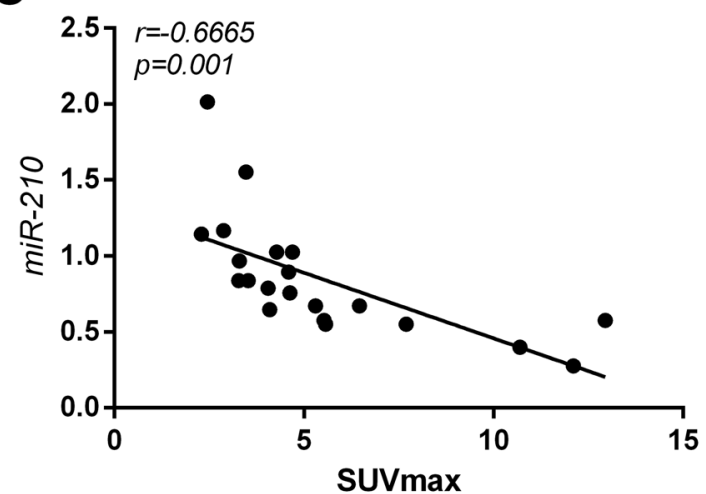

$\mathbf{E}$

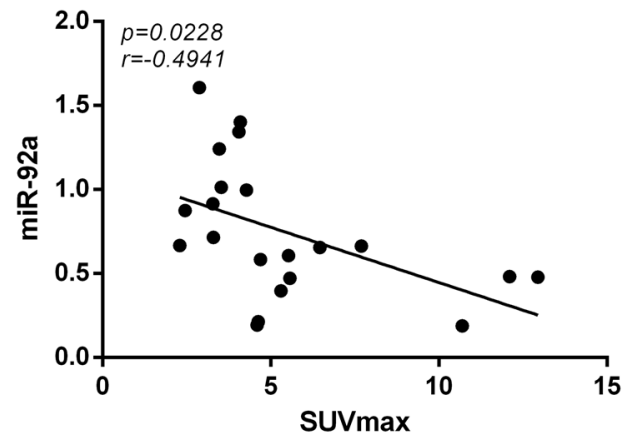

B

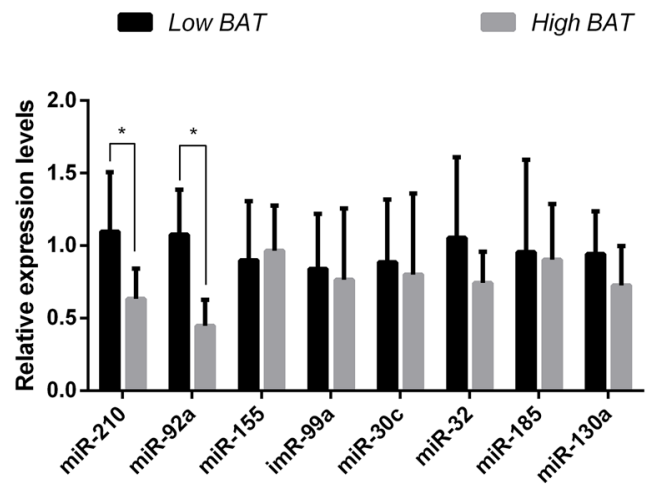

D

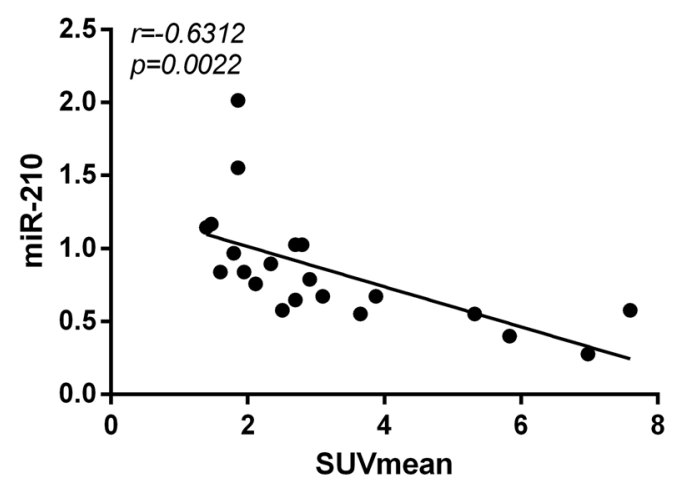

$\mathbf{F}$

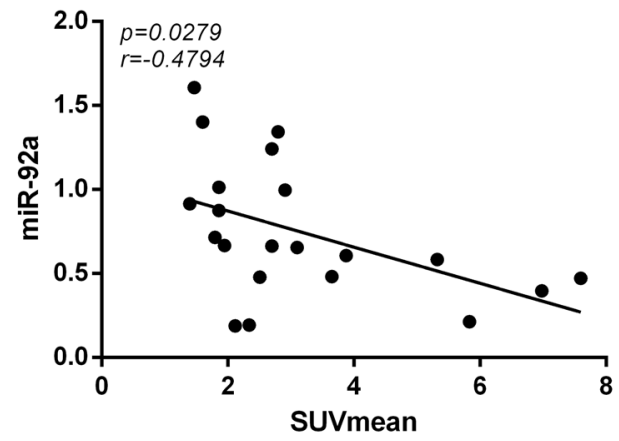

Figure 5. (A) Mice serum exosomal miRNA expression profiles in the 2,300 $\mathrm{m}$ and 4,300 $\mathrm{m}$ groups are shown in the volcano plots as the log transformed fold up- and downregulation values; exosomal miRNAs for which $-1<\log 2$ (fold change) $>1$ and $-\log 10$ ( $\mathrm{p}$ value) $>1.303$ were considered significantly differentially expressed. (B) Human serum exosomal miRNAs were detected in the high-BAT and low-BAT groups, and the relative quantification was obtained by the $\Delta \Delta \mathrm{CT}$ method with normalization to $\mathrm{U} 6$ ( $\mathrm{n}=21$ per group, ${ }^{*} \mathrm{p} \leq 0.05$ indicates statistical significance). The low-BAT group was the control group. (C-F) The relationship between the relative expression level of miR-210 or miR-92a in human serum exosomes ( $\triangle \Delta C T$ method vs. U6) and ${ }^{18} \mathrm{~F}-\mathrm{FDG}$ PET/CT brown adipose SUVmax/mean values (Pearson correlation; $\mathrm{n}=21 ; \mathrm{p} \leq 0.05$ indicates statistical significance).

mitochondrial uncoupling. Collectively, the above results supported our hypothesis. BAT dissipates energy, maintains thermoneutrality, and promotes insulin sensitivity; moreover, cold exposure activates BAT in adults (as measured by glucose uptake ${ }^{26}$ and reduces the risk of obesity and diabetes. Therefore, BAT might contribute to the promising effects of high altitude. 


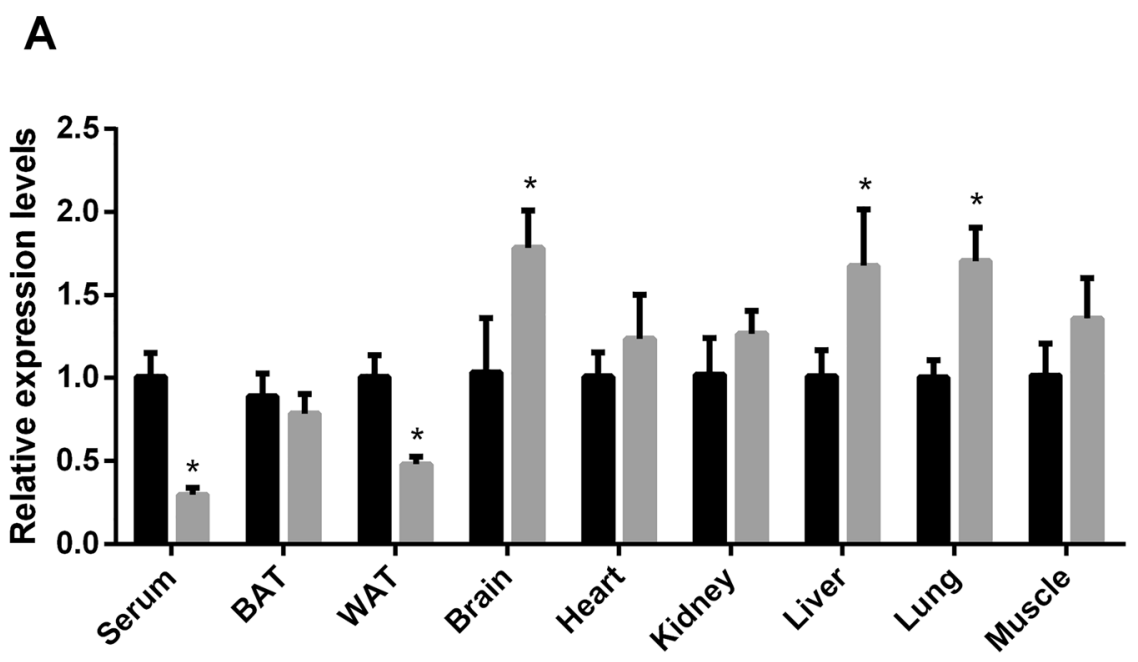

B

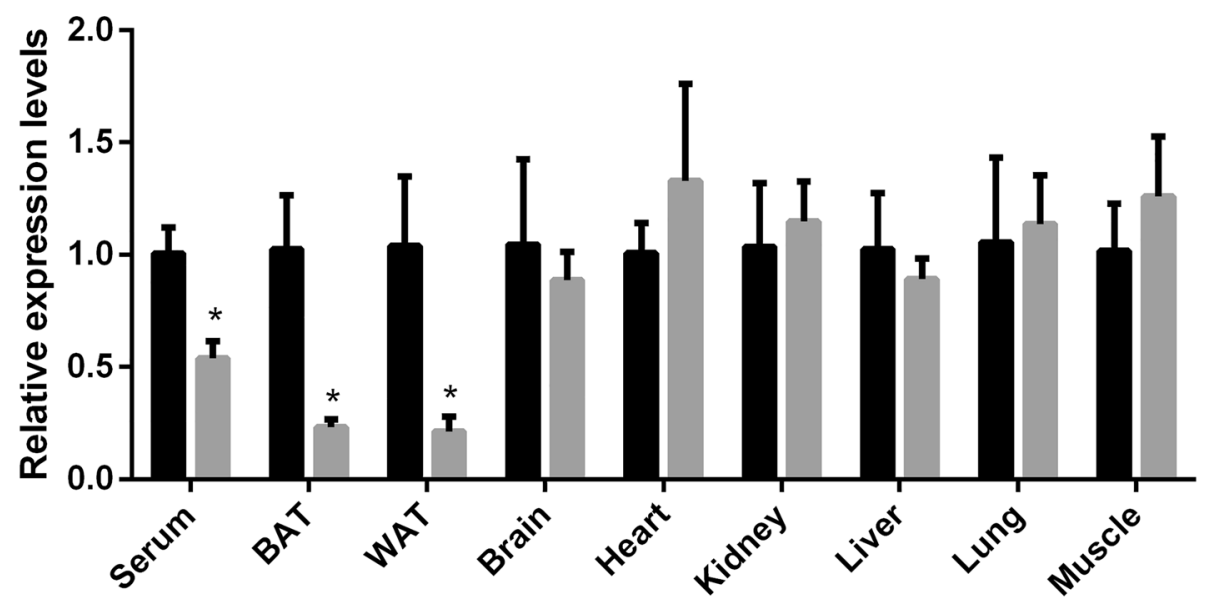

Figure 6. The relative expression levels of miR-210/92a in different tissues of 2,300 m- and 4,300 m-raised mice. (A) The relative expression levels of miR-210. (B) The relative expression levels of miR-92a. The relative quantification was obtained by the $\Delta \Delta$ CT method with normalization to U6. $\mathrm{n}=6$ per group; ${ }^{*} \mathrm{p}<0.05$ indicates statistical significance. The 2,300 $\mathrm{m}$ group was used as the control.

The present study demonstrated changes in BAT levels in humans and mice caused by exosomal miRNA expression levels. In addition, the expression levels of WAT-derived exosomal miRNAs were the same to serum, after injected with miRNAs-angomirs. Interestingly, Ying et al. reported that adipose tissues can release an abundance of exosomes that crosstalk with macrophages in both the remote liver and neighboring adipose tissue to affect insulin sensitivity, thus regulating metabolic disease ${ }^{27}$. Moreover, WAT-derived exosomes have already been demonstrated to be powerful cell-cell communication vehicles via their cargo of exosomal miRNAs, which interfere with global metabolism ${ }^{28}$. In addition, WAT plays an important role in mediating miRNA expression levels by manipulating Dicer activity, and many exosomal miRNAs have been found to be differentially expressed in lean and obese individuals, and these miRNAs have been demonstrated to originate from WAT ${ }^{29}$. A few previous studies indicated miRNAs can be packaged into and transported via exosomes, have profound effects on BAT activity or as biomarkers, Such as miR-103, miR-146b, miR-155 and miR-99a ${ }^{30}$. This finding is consistent with our results showing similar expression in exosomes, but these results were not seen in the mouse models in this study, possibly because they are not hypoxia-sensitive or are not affected by hypobaric hypoxia. However, in contrast, our study showed elevated BAT activity accompanied by significantly decreased expression levels of miR-210 and miR-92a.

miR-210 is a specific target of Hif- $1 \alpha^{31}$ and exosomal miR-210 from hypoxic cells can be transferred to various types of recipient cells and involved in various processes, including the cell cycle, mitochondrial oxidative metabolism, angiogenesis, the DNA damage response, and cell survival ${ }^{32}$. However, few studies have shown 
- Ctrl $\square$ ncRNA $\square$ miR-210-agomir miR-92a-agomir $\square$ miR-210/92a-agomir

A

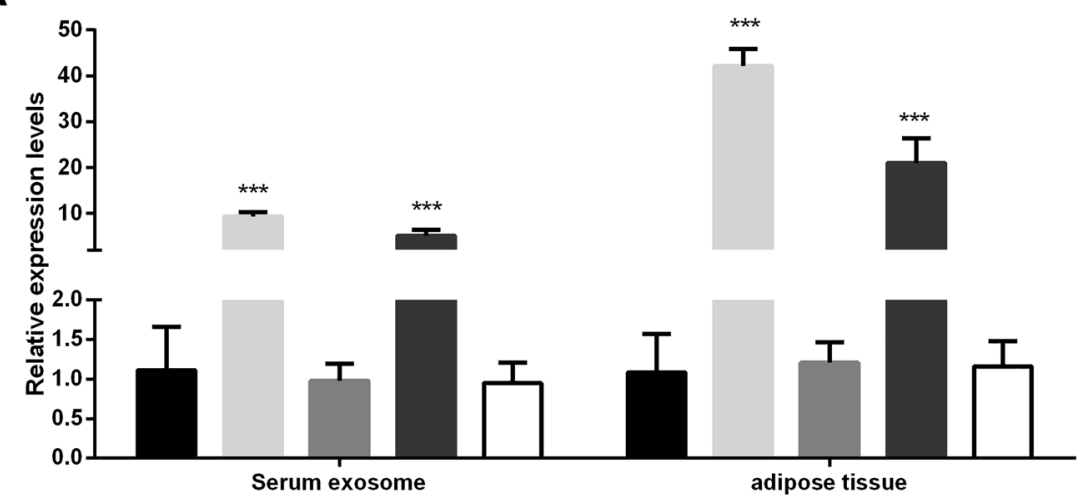

B
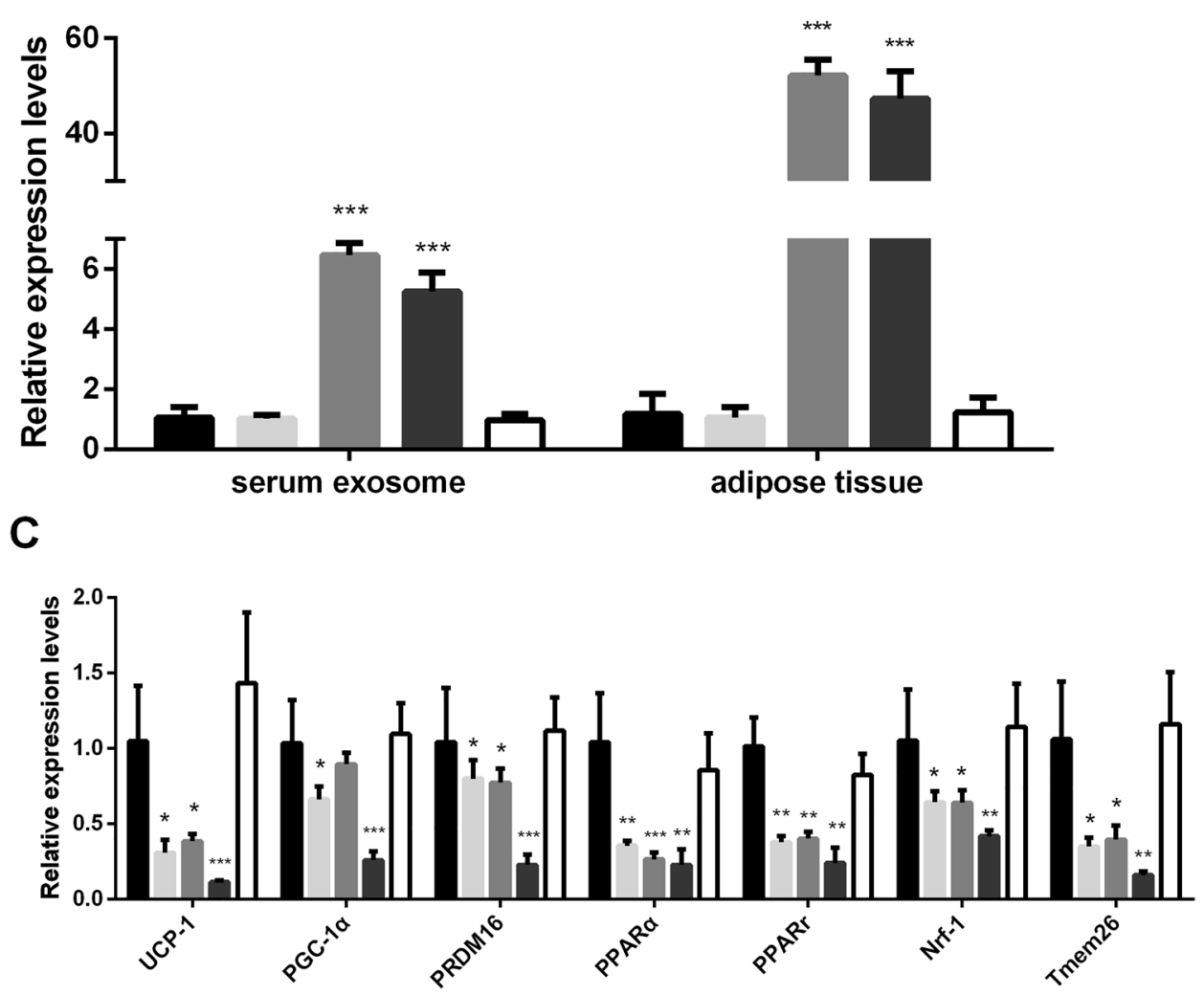

Figure 7. (A) The relative expression levels of miR-210 in serum and visceral WAT from the agomir/ncRNA/ Ctrl groups of 4,300 m-raised mice. (B) The relative expression levels of miR-92a in serum and visceral WAT from the agomir/ncRNA/Ctrl groups of 4,300 m-raised mice. The results were obtained by qPCR analysis. The relative quantification was obtained by the $\Delta \Delta$ CT method with normalization to U6. $n=6$ per group. (C) Brown adipose-selective genes' relative expression levels in BAT from the agomir/ncRNA/Ctrl groups of 4,300 m-raised mice. The relative quantification was obtained by the $\Delta \Delta \mathrm{CT}$ method compared with $18 \mathrm{~S}$ rRNA. $\mathrm{n}=6$ per group $\left({ }^{*} \mathrm{p} \leq 0.05\right.$ indicates statistical significance. ${ }^{* *} \mathrm{p} \leq 0.01,{ }^{* * *} \mathrm{p} \leq 0.001$, Student's $t$ test. The Ctrl- $(4,300 \mathrm{~m})$ group was used as the control.

the expression and function of Hif- $\alpha$ in tissues under long-term exposure to environmental hypoxia. In this study, we found that miR-210 levels were significantly decreased in the high-BAT group compared with the low-BAT group. Similar results were also observed in mice exposed to chronic hypoxia, in which the activity of mitochondria in BAT indicated that oxygen consumption increased in complex I activity. In addition, the expression levels of Hif- $1 \alpha$ in WAT has suppressed significantly after 8 weeks exposure to hypobaric hypoxia. Furthermore, miR-210 had significantly lower in the serum- and WAT-derived exosomes of mice after longterm hypoxia exposure. which can be attributed to the unique hypoxia-sensing and HIF signaling pathways and exosome secretion mechanisms in adipose tissues. Our study also showed that Fgfr-1 in BAT has increased 
A

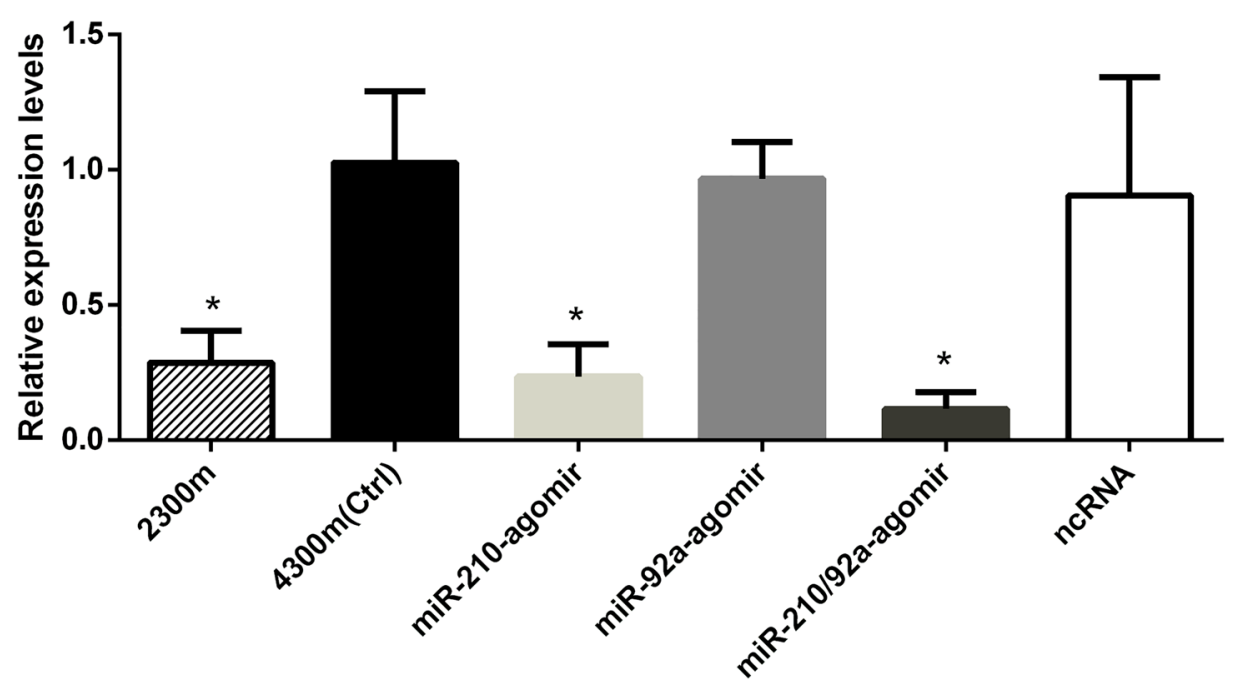

B

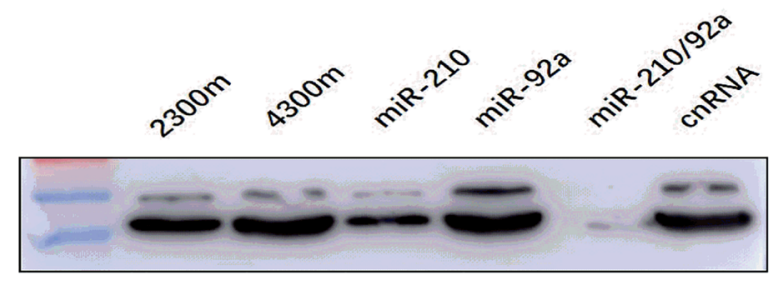

FGFR-1 (120kDa)

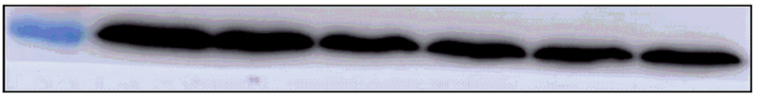

$\alpha-T u b u l i n(55 k D a)$

C

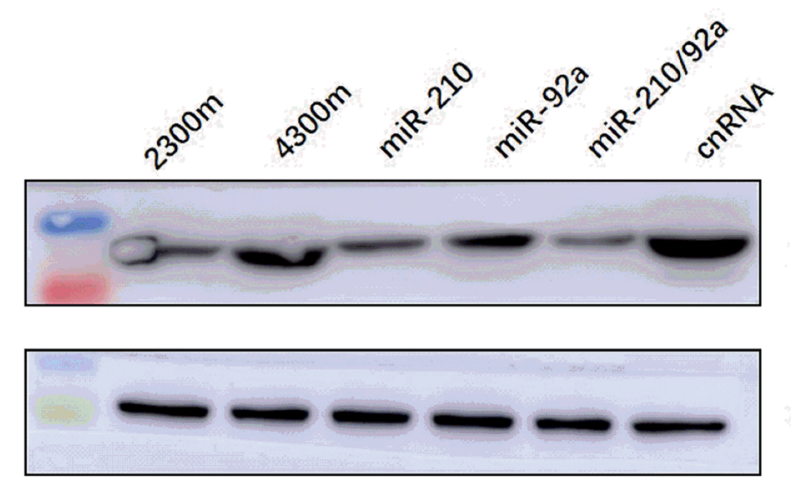

UCP-1(32kDa)

$\alpha-T u b u l i n(55 \mathrm{kDa})$

Figure 8. (A) The relative expression levels of Fgfr-1 in BAT from the agomir/ncRNA/Ctrl groups of 4,300 mand 2,300 m-raised mice. The results were obtained by qPCR analysis. The relative quantification was obtained by the $\Delta \Delta$ CT method with normalization to $18 \mathrm{~S}$ rRNA. $\mathrm{n}=18$; ${ }^{\star} \mathrm{p} \leq 0.05$, Student's $t$ test. The Ctrl- $(4,300 \mathrm{~m})$ group was used as the control. $(\mathbf{B}, \mathbf{C})$ The protein expression levels of FGFR-1/UCP-1 in BAT from the agomir/ ncRNA/Ctrl groups of 4,300 $\mathrm{m}$ - and $2300 \mathrm{~m}$-raised mice.

significantly in 4,300 m mice, but after manipulatively increased the miR-210 by locally injected agomir, Fgfr-1 have noticeably suppressed. Due to effective stimulator for improving BAT activity, FGF-21 is a critical protein to influence on metabolic homeostasis ${ }^{33}$. FGFR-1, the most affinity receptor with FGF-21, is a family member of the tyrosine kinase receptors FGFR isoforms, normally expressed on WAT, BAT and hypothalamus ${ }^{34}$. Based on the previous reports ${ }^{35}$, FGF-21/FGFR-1 signals induced BAT activity majorly caused by regulations of PGC-1a and UCP-1 pathway. Moreover, FGFR-1, a major target downstream, can be negatively modulated by miR- $210^{23}$. FGFR-1 played important role in hypoxia microenvironment, with involved in development, proliferation, and angiogenesis in adipocytes ${ }^{36}$. Consistently with the current study, BAT activity was enhanced by specifically promoted FGFR-1 on mice adipose tissues ${ }^{36}$, but Fgfr-1 KO mice shown the opposite effects ${ }^{37}$. Totally, this study 
A

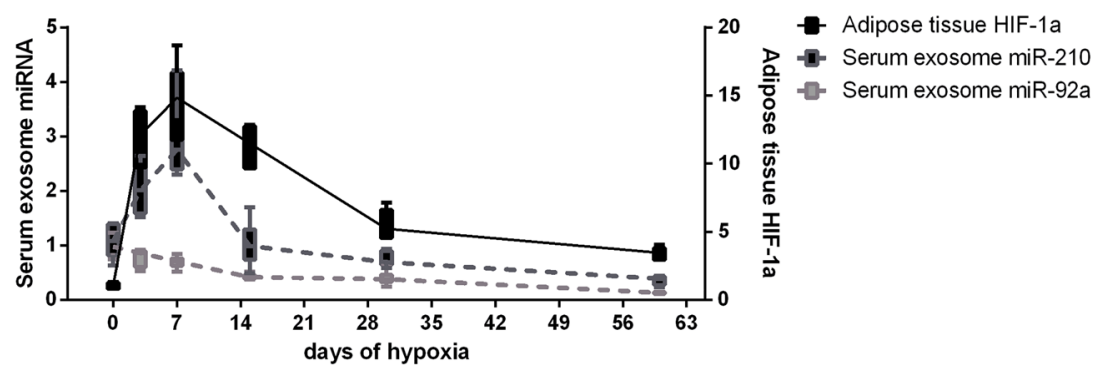

B

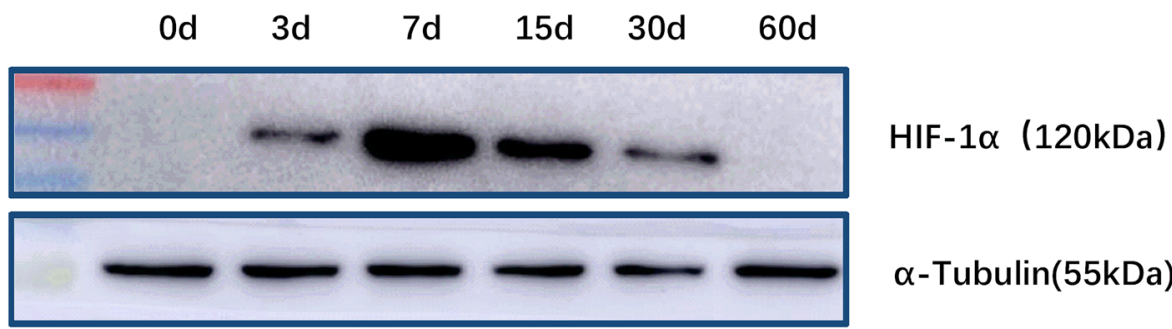

Figure 9. (A) Dynamic variation in the relative expression level of Hif- $1 \alpha$ in visceral WAT and the serum exosomal miR-210/92a with the duration of hypoxia. The left $\mathrm{Y}$ axis indicates the relative expression of miR210/92a, and the right $Y$ axis indicates the relative expression of Hif-1a. (B) The dynamic variation in the protein expression levels of HIF- $1 \alpha$ as determined by Western blot analysis.

indicated that high-altitude hypoxia activated BAT through attenuated WAT secreted exosomal miR-210, which enhanced the FGFR-1 expression in BAT.

Interestingly, we found that the expression level of FGFR-1 in BAT was not changed, but UCP-1 expression was significantly decreased, in the 4,300 m raised mice with miR-92a-agomir-injected groups. Furthermore, the expression level of FGFR-1 was decreased more obviously in the miR-210/92a agomir-injected groups compared with the group injected with the miR-210 agomir alone. It indicated that miR-92a increased the inhibitory effect of miR-210 on FGFR-1, miR-210/92a cluster synergistically increased the inhibition of BAT activity. Furthermore, miR-92a did not directly decrease BAT activity through the FGFR-1 pathway and that other mechanisms may be involved.

Notably, this study showed that the expression level of serum exosomal miR-92a increased in subjects with high BAT and in mice raised under hypoxic conditions. Similarly, miR-92a is an adipose-derived miRNA and exhibited low expression levels in serum exosome and WAT from high-altitude-raised mice. The significant biomarker for BAT was miR-92, according to the evidence from Chen $\mathrm{Y}^{38}$ together with the angiogenesis-modulating effects of miR-92a in adipose tissues. Decreased miR-92a expression might promote angiogenesis in BAT after hypoxia, resulting in enhanced blood supply to local adipose tissues followed by an increase in browning activity. Furthermore, the similar responses of miR-92a and miR-210 after exposure to hypoxia might indicate the involvement of miR-92a in both hypoxic and metabolic signaling.

Our study has some limitations. First, we conduct PET/CT scans only in high-altitude populations. We utilized all subjects to study exposure to hypoxia at different altitudes without the sea level control group. This methodology could have influenced the BAT results. Furthermore, no data are available for sea level human populations or mice raised at sea level. Second, the small sample size for the collection of PET/CT data was due to insufficient funding along with expensive testing fees and radiation exposure risks of PET/CT to healthy people in the clinic. Finally, the specific genetic manipulations of miRNAs have not been validated at the cellular level. Such validation needs to be conducted in future studies.

\section{Methods}

Study approval. The human study was approved by the Ethics Committees of Qinghai University (No. QHUM-20171101HM) and Qinghai Provincial People's Hospital (QHPH-20171122). All subjects provided written informed consent prior to any study procedure. All procedures were conducted in accordance with the principles of the Declaration of Helsinki.

Statistics. General parameters for subject characteristics are presented as the mean \pm s.d. Statistical differences were assessed by applying Student's $t$ test (unpaired, two-tailed). Pearson correlation was used in correla- 


\begin{tabular}{|l|c|c|c|l|}
\hline & Total $(\mathbf{n}=\mathbf{4 0})$ & $\mathbf{2 , 3 0 0} \mathbf{~ m}(\mathbf{n}=\mathbf{2 0})$ & $\mathbf{4 , 3 0 0} \mathbf{~ m}(\mathbf{n}=\mathbf{2 0})$ & $\mathbf{p}$ \\
\hline Weight $(\mathrm{g})$ & $25.12 \pm 1.65$ & $26.03 \pm 1.5$ & $24.22 \pm 1.3$ & $0.000^{*}$ \\
\hline RBC $\left(10^{12} / \mathrm{l}\right)$ & $8.91 \pm 0.837$ & $8.78 \pm 0.87$ & $9.08 \pm 0.79$ & 0.355 \\
\hline HGB $(\mathrm{g} / \mathrm{l})$ & $140 \pm 13.6$ & $133 \pm 10.7$ & $150 \pm 10.5$ & $0.000^{*}$ \\
\hline HCT\% & $43.2 \pm 4.19$ & $41.3 \pm 3.76$ & $45.6 \pm 3.5$ & $0.000^{*}$ \\
\hline WBC $\left(10^{9} / \mathrm{l}\right)$ & $3.68 \pm 2.14$ & $3.04 \pm 1.61$ & $4.47 \pm 2.31$ & 0.086 \\
\hline
\end{tabular}

Table 2. The general parameters of mice data.

tion analysis. GraphPad Prism 6 or Excel software (Microsoft Office) was applied to calculate p values. The level of statistical significance was set at $\mathrm{p} \leq 0.05$.

PET/CT scanning and baseline measurements. We recruited 21 individuals of Han Chinese descent living at different altitudes on the Qinghai-Tibet Plateau. The details are shown in Supplement Table 1. From each volunteer, after $8 \mathrm{~h}$ of fasting, $15 \mathrm{ml}$ of venous whole blood was collected for the measurement of serum levels of triglycerides (TG), cholesterol (CHOL), high-density lipoprotein cholesterol (HDL-C), low-density lipoprotein cholesterol (LDL-C), and so on. Serum samples were isolated within $1 \mathrm{~h}$, and exosomes were then purified from each serum sample. The general features of exosomes isolated from serum in the current study were confirmed by FACS analysis of surface CD63 and TEM, as reported previously. Prior to PET/CT scanning, the height and weight, along with the waist and hip circumference, were measured. After $8 \mathrm{~h}$ of fasting, ${ }^{18} \mathrm{~F}-\mathrm{FDG}$ was injected intravenously at a dose of $0.1 \mathrm{mCi}$ per kilogram of body weight. A full-body PET/CT scan was performed after a rest period at $4{ }^{\circ} \mathrm{C}$ for $60 \mathrm{~min}$. BAT activity was measured from static PET scans as the mean and maximal ${ }^{18} \mathrm{~F}-\mathrm{FDG}$ uptake in BAT. The investigated regions were manually outlined using a threshold of 1.5 SUV and Hounsfield units between -10 and -180 to define BAT. The metabolic and PET characteristics of the recruited human subjects are shown in Table 1.

Animal models. Six-week-old male C57BL/6J mice were housed in a temperature-controlled environment on a 12:12 h light: dark cycle at altitudes of $2,300 \mathrm{~m}$ and 4,300 $\mathrm{m}$, separately. The temperature and humidity were controlled to remain within $22-25^{\circ} \mathrm{C}$ and $50-60 \%$, respectively. The water and food supply were unrestricted. To determine the influence in serum exosomal miRNA and adipose tissue HIF-1 $\alpha$ expression that may occur at long-term hypoxia exposure, mice were euthanized at six different stages (hypoxia exposure day 0, 3, 7, 15, 30 and 60). And then the serum and tissues were taken and frozen in liquid nitrogen within $30 \mathrm{~s}$ for the next study. For in vivo experiments, we delivered miR-210 or miR-92a agomirs ( $20 \mathrm{nmol} / \mathrm{mice})$ or scrambled negative control sequences (GenePharma, Shanghai, China) to the 4,300 m-raised mice though inguinal subcutaneous fat pad injection. The injections were performed four times at three-day intervals. After the final injection, the mice were housed under the same conditions described above. Four weeks after the final injection, serum and tissues were collected for the next experiment. All experiments were conducted in strict accordance with the National Institutes of Health Guide for the Use of Laboratory Animals. All efforts were made to minimize suffering. The general parameters of mice data are shown in Table 2.

Exosome isolation, fixation, and identification. Venous blood was collected from humans and mice, and serum was separated for the next experiment. Fresh tissue materials (100 mg), such as adipose, liver, heart, and brain tissue, were split into small pieces and soaked in $1 \mathrm{ml}$ of PBS. Samples were incubated for $4 \mathrm{~h}$ at room temperature for complete exosome release, and the PBS was then collected for the next experiment ${ }^{27}$.

A total of $250 \mu \mathrm{l}$ of murine or human serum and $1 \mathrm{ml}$ of exosome-rich PBS were isolated using ExoQuick exosome precipitation solution (\#: EXOQ5A-1, SBI) following the manufacturer's protocol. For exosome loading, exosome preparations were isolated and diluted with PBS to a final volume of $100 \mu \mathrm{l}$ and prepared for electron microscopy and other experiments. The exosomes were resuspended in 50-100 $\mu \mathrm{l}$ of $2 \%$ PFA, and $5 \mu \mathrm{l}$ of the exosomal suspension was added to a Formvar/carbon-loaded copper mesh and washed with PBS. In all steps, the Formvar membrane was kept moist, and the other side was kept dry. The copper mesh was placed on $50 \mu \mathrm{l}$ of $1 \%$ glutaraldehyde droplets for $5 \mathrm{~min}$ and washed 8 times in $100 \mu \mathrm{l}$ of ultrapure water for $5 \mathrm{~min}$. The copper mesh carrying the exosomes was placed on $50 \mu \mathrm{l}$ of uranyl oxalate droplets ( $\mathrm{pH} 7$ ) for 5 min and then on $50 \mu \mathrm{l}$ of methylcellulose droplets for $10 \mathrm{~min}$. The excess liquid on the filter paper was aspirated, and the mesh was dried in air for 5-10 min. All of the above operations were carried out on ice. Electron micrographs were acquired at $80 \mathrm{kV}$. To determine the exosomal particle sizes, the concentration was assessed using NTA. An NTA device (ZetaView , Particle Metrix, Germany) was used to measure the particle size, concentration, and zeta potential of exosomes by analyzing the particle traces. To evaluate exosome marker proteins, exosomes were lysed with RIPA buffer, and total protein was separated by SDS-PAGE using $12 \%$ polyacrylamide gels and transferred to a PVDF membrane, which was blocked with $5 \%$ BSA for $1 \mathrm{~h}$ and incubated with primary antibodies at $4{ }^{\circ} \mathrm{C}$ overnight. Antibody binding was detected with a chemiluminescence reagent (Thermo Fisher Scientific). Antibodies included rabbit anti-CD9 (\#: ab 92726, Abcam), diluted 1:2000; rabbit anti-CD63 (\#: ab 217345, Abcam), diluted 1:1,000; and goat anti-rabbit IgG (\#: SA00001-2, Proteintech), diluted 1:1,000. 


\begin{tabular}{|c|c|}
\hline mRNAs/miRNAs & $5^{\prime} \rightarrow 3^{\prime}$ \\
\hline \multicolumn{2}{|l|}{$18 \mathrm{~S}$} \\
\hline Forward & TTGACGGAAGGGCACCACCAG \\
\hline Reverse & GCACCACCACCCACGGAATCG \\
\hline \multicolumn{2}{|l|}{ Ucp-1 } \\
\hline Forward & CAGAGGTCGTGAAGGTCAGAATGC \\
\hline Reverse & GGACATCGCACAGCTTGGTACG \\
\hline \multicolumn{2}{|l|}{ Ppargcla } \\
\hline Forward & CCGAAGACACTACAGGTTCCATAG \\
\hline Reverse & GGGAGGGAGAGAGGAGAGAGG \\
\hline \multicolumn{2}{|l|}{ Prdm16 } \\
\hline Forward & CAACAAAGAGAAGCCGTTCAAG \\
\hline Reverse & TTTCGGATCTCGGAGAAGTAAG \\
\hline \multicolumn{2}{|l|}{ Ppara } \\
\hline Forward & GAGCTGCAAGATTCAGAAGAAG \\
\hline Reverse & GAATCTTTCAGGTCGTGTTCAC \\
\hline \multicolumn{2}{|l|}{ Ppary } \\
\hline Forward & TGTGGACCTCTCCGTGATGG \\
\hline Reverse & GGTTCTACTTTGATCGCACTTTGG \\
\hline \multicolumn{2}{|l|}{ Nrf1 } \\
\hline Forward & CCACGTTGGATGAGTACACG \\
\hline Reverse & GCACCACATTCTCCAAAGGT \\
\hline \multicolumn{2}{|l|}{ Tfam } \\
\hline Forward & TCAGGAGCAGCAGGCACTACA \\
\hline Reverse & CTGAGCTCCGAGTCCTTGAACAC \\
\hline \multicolumn{2}{|l|}{ Tmem26 } \\
\hline Forward & GTGGTGTGGACGTGGAGTATGC \\
\hline Reverse & GTAGATCATCAGGACGAGGCGC \\
\hline \multicolumn{2}{|l|}{ Hif- $1 a$} \\
\hline Forward & CCACCACAACTGCСACСACTG \\
\hline Reverse & TGCCACTGTATGCTGATGCCTTAG \\
\hline \multicolumn{2}{|l|}{ Fgfr-1 } \\
\hline Forward & TTGCCAGACCAAGAAGAAGCCATG \\
\hline Reverse & GTCCTTGTCACCACTGCGTTCTC \\
\hline \multicolumn{2}{|c|}{ has-/mmu-miR-210-3p } \\
\hline Forward & CUGUGCGUGUGACAGCGGCUGA \\
\hline \multicolumn{2}{|c|}{ has-/mmu-miR-92a-3p } \\
\hline Forward & UAUUGCACUUGUCCCGGCCUG \\
\hline \multicolumn{2}{|l|}{ hsa-miR-155-5p } \\
\hline Forward & UUAAUGCUAAUUGUGAUAGGGGU \\
\hline \multicolumn{2}{|l|}{ hsa-miR-99a-5p } \\
\hline Forward & AACCCGUAGAUCCGAUCUUGUG \\
\hline \multicolumn{2}{|l|}{ has-miR-30c-5p } \\
\hline Forward & UGUAAACAUCCUACACUCUCAGC \\
\hline \multicolumn{2}{|l|}{ hsa-miR-32-3p } \\
\hline Forward & CAAUUUAGUGUGUGUGAUAUUU \\
\hline \multicolumn{2}{|l|}{ hsa-miR-185-5p } \\
\hline Forward & UGGAGAGAAAGGCAGUUCCUGA \\
\hline \multicolumn{2}{|l|}{ hsa-miR-130a-3p } \\
\hline Forward & CAGUGCAAUGUUAAAAGGGCAU \\
\hline
\end{tabular}

Table 3. Primer sequences for genes.

mRNA/miRNA PCR and MiFinder PCR array. mRNA was isolated from mouse tissues using an RNAsimple Total RNA Isolation Kit (\#: DP419, TIANGEN China Beijing). Reverse transcription and fluorescence quantitative PCR of tissue mRNA were performed with FastKing gDNA Dispelling RT SuperMix (\#: 
KR118, TIANGEN China Beijing) and a SuperReal PreMix Kit (SYBR Green) (\#: FP215, TIANGEN China Beijing).

For isolation of serum or tissue exosomal miRNA, 400-600 $\mu \mathrm{l}$ of exosome extract was obtained from mice or humans, and miRNAs were isolated using a SeraMir Exosome RNA Purification Kit (\#: RA806A-1, SBI) following the manufacturer's protocol. Reverse transcription and fluorescence quantitative PCR of all miRNA samples was performed with a miRcute Enhanced miRNA cDNA First Chain Synthesis Kit (\#: KR211, TIANGEN China Beijing) and a miRcute Enhanced miRNA Fluorescence Quantification Kit (SYBR Green) with reverse universal primers (\#: FP411, TIANGEN China Beijing). A miScript ${ }^{\mathrm{Tm}}$ miRNA PCR Array Human Hypoxia Signaling (\#: MIHS-121Z QIAGEN Germany) was analyzed according to the manufacturer's protocol. The primer sequences are provided in Table 3.

Western blot analysis and histomorphology. The method used for Western blot analysis of tissue proteins was the same as that used for exosome markers. Antibodies included rabbit anti-UCP-1 (\#: ab10983, Abcam), diluted 1:5,000; rabbit anti-FGFR-1 (\#: 9740, CST), rabbit anti-HIF-1a (\#: 36169, CST), $\alpha$-Tubulin Rabbit Polyclonal Antibody (\#: AF0001, Beyotime), diluted 1:1,000; and goat anti-rabbit IgG (\#: SA00001-2, Proteintech),diluted 1:1,000. Immunohistochemical staining was performed according to standard methods. The antibody used was rabbit anti-UCP-1 (\#: ab10983, Abcam), diluted 1:500.

Mitochondrial respiration assay. BAT mitochondrial respiration rates were determined by measuring the OCR with a high-resolution mitochondrial respirometer (Oxygraph-2k). In brief, $5 \mathrm{mg}$ of pure adipose tissue was isolated in $500 \mu \mathrm{l}$ of precooled MiR05 respirator $(110 \mathrm{mM}$ sucrose, $60 \mathrm{mM}$ K-lactobionate, $0.5 \mathrm{mM}$ EGTA, $3 \mathrm{mM} \mathrm{MgCl}_{2}, 20 \mathrm{mM}$ taurine, $10 \mathrm{mM} \mathrm{KH}_{2} \mathrm{PO}_{4}, 20 \mathrm{mM}$ HEPES ( $\mathrm{pH} 7.1$ ) and $0.1 \% \mathrm{BSA}$ at $30{ }^{\circ} \mathrm{C}$, homogenized, and added to the chamber of the high-resolution mitochondrial respirometer. The temperature in the chamber was maintained at $37^{\circ} \mathrm{C}$. Pyruvate $(5 \mathrm{mM})$, malic acid $(5 \mathrm{mM})$, and sodium glutamate $(10 \mathrm{mM})$ were used to measure the respiration rate according to substrate-uncoupler-inhibitor-titration (SUIT) protocols. After baseline stabilization, the state 4 respiration rate (CI-LEAK) of respiratory chain complex I was obtained. ADP $(10 \mathrm{mM})$, cytochrome C $(10 \mu \mathrm{M})$, succinic acid $(10 \mathrm{mM})$, FCCP $(0.25-1.5 \mu \mathrm{M})$, and rotenone $(0.5 \mu \mathrm{M})$ were sequentially added. The mitochondrial respiratory chain complex I state 3 respiration rate (CI-OXPHOS), respiratory chain complexes I + II state 3 respiration rate $(\mathrm{CI}+\mathrm{II}-\mathrm{OXPHOS})$, and electron transport capacity (CI+ II-ETS), as well as the electron transport capacity (CII-ETS) and uncoupling ability of respiratory chain complex II were measured under the condition of intact mitochondrial inner membranes.

Received: 24 June 2019; Accepted: 12 December 2019

Published online: 01 September 2020

\section{References}

1. Hodson, L., Humphreys, S. M., Karpe, F. \& Frayn, K. N. Metabolic signatures of human adipose tissue hypoxia in obesity. Diabetes 62, 1417-1425. https://doi.org/10.2337/db12-1032 (2013).

2. Bigham, A. W. \& Lee, F. S. Human high-altitude adaptation: forward genetics meets the HIF pathway. Genes Dev. 28, $2189-2204$. https://doi.org/10.1101/gad.250167.114 (2014).

3. Bigham, A. W. Genetics of human origin and evolution: high-altitude adaptations. Curr. Opin. Genet. Dev. 41, 8-13. https://doi. org/10.1016/j.gde.2016.06.018 (2016).

4. Lorenzo, F. R. et al. A genetic mechanism for Tibetan high-altitude adaptation. Nat. Genet. 46, 951-956. https://doi.org/10.1038/ ng.3067 (2014).

5. Petousi, N. \& Robbins, P. A. Human adaptation to the hypoxia of high altitude: the Tibetan paradigm from the pregenomic to the postgenomic era. J. Appl. Physiol. 116, 875-884. https://doi.org/10.1152/japplphysiol.00605.2013 (2014).

6. D’Alessandro, A. et al. AltitudeOmics: red blood cell metabolic adaptation to high altitude hypoxia. J. Proteome Res. 15, 3883-3895. https://doi.org/10.1021/acs.jproteome.6b00733 (2016).

7. Horscroft, J. A. et al. Metabolic basis to Sherpa altitude adaptation. Proc. Natl. Acad. Sci. U.S.A. 114, 6382-6387. https://doi. org/10.1073/pnas.1700527114 (2017).

8. Murray, A. J. \& Horscroft, J. A. Mitochondrial function at extreme high altitude. J. Physiol. 594, 1137-1149. https://doi.org/10.1113/ JP270079 (2016)

9. Murray, A. J. Energy metabolism and the high-altitude environment. Exp. Physiol. 101, 23-27. https://doi.org/10.1113/ep085317 (2016).

10. Ge, R. L., Simonson, T. S., Gordeuk, V., Prchal, J. T. \& McClain, D. A. Metabolic aspects of high-altitude adaptation in Tibetans. Exp. Physiol. 100, 1247-1255. https://doi.org/10.1113/EP085292 (2015).

11. Xu, S. et al. The prevalence of and risk factors for diabetes mellitus and impaired glucose tolerance among Tibetans in China: a cross-sectional study. Oncotarget 8, 112467-112476. https://doi.org/10.18632/oncotarget.21301 (2017).

12. Shen, X., Vaidya, A., Wu, S. \& Gao, X. The diabetes epidemic in china: an integrated review of national surveys. Endocr. Pract. 22, 1119-1129. https://doi.org/10.4158/ep161199.Ra (2016).

13. Grundy, S. M. Obesity, metabolic syndrome, and cardiovascular disease. J. Clin. Endocrinol. Metab. 89, 2595-2600. https://doi. org/10.1210/jc.2004-0372 (2004).

14. Jankovic, A. et al. Two key temporally distinguishable molecular and cellular components of white adipose tissue browning during cold acclimation. J. Physiol. 593, 3267-3280. https://doi.org/10.1113/jp270805 (2015).

15. Bai, Z. et al. Intermittent cold exposure results in visceral adipose tissue "browning" in the plateau pika (Ochotona curzoniae). Comp. Biochem. Physiol. A Mol. Integr. Physiol. 184, 171-178. https://doi.org/10.1016/j.cbpa.2015.01.019 (2015).

16. Li, J. et al. Chronic cold exposure results in subcutaneous adipose tissue browning and altered global metabolism in Qinghai-Tibetan plateau pika (Ochotona curzoniae). Biochem. Biophys. Res. Commun. 500, 117-123. https://doi.org/10.1016/j. bbrc.2018.03.147 (2018).

17. Paolicelli, R. C., Bergamini, G. \& Rajendran, L. Cell-to-cell communication by extracellular vesicles: focus on microglia. Neuroscience 405, 148-157. https://doi.org/10.1016/j.neuroscience.2018.04.003 (2019). 
18. Zhang, D., Lee, H., Zhu, Z., Minhas, J. K. \& Jin, Y. Enrichment of selective miRNAs in exosomes and delivery of exosomal miRNAs in vitro and in vivo. Am. J. Physiol. Lung Cell. Mol. Physiol. 312, L110-L121. https://doi.org/10.1152/ajplung.00423.2016 (2017).

19. Nader, J. et al. miR-92a: a novel potential biomarker of rapid aortic valve calcification. J. Heart Valve Dis. 26, 327-333 (2017).

20. Hong, T. S. et al. Brown adipose tissue ${ }^{18} \mathrm{~F}-\mathrm{FDG}$ uptake in pediatric PET/CT imaging. Pediatr. Radiol. 41, 759-768. https://doi. org/10.1007/s00247-010-1925-y (2011).

21. Tsuchiya, S. et al. MicroRNA-210 regulates cancer cell proliferation through targeting fibroblast growth factor receptor-like 1 (FGFRL1). J. Biol. Chem. 286, 420-428. https://doi.org/10.1074/jbc.M110.170852 (2011).

22. Chang, W. et al. Survival of hypoxic human mesenchymal stem cells is enhanced by a positive feedback loop involving miR-210 and hypoxia-inducible factor 1. J. Vet. Sci. 14, 69-76 (2013).

23. Yang, Y. et al. MicroRNA-210 promotes cancer angiogenesis by targeting fibroblast growth factor receptor-like 1 in hepatocellular carcinoma. Oncol. Rep. 36, 2553-2562. https://doi.org/10.3892/or.2016.5129 (2016).

24. Huang, X. \& Zuo, J. Emerging roles of miR-210 and other non-coding RNAs in the hypoxic response. Acta Biochim. Biophys. Sin. 46, 220-232. https://doi.org/10.1093/abbs/gmt141 (2014).

25. Dünnwald, T., Gatterer, H., Faulhaber, M., Arvandi, M. \& Schobersberger, W. Body composition and body weight changes at different altitude levels: a systematic review and meta-analysis. Front. Physiol. 10, 1-24. https://doi.org/10.3389/fphys.2019.00430 (2019).

26. Sidossis, L. \& Kajimura, S. Brown and beige fat in humans: thermogenic adipocytes that control energy and glucose homeostasis. J. Clin. Investig. 125, 478-486. https://doi.org/10.1172/JCI78362 (2015).

27. Ying, W. et al. Adipose tissue macrophage-derived exosomal miRNAs can modulate in vivo and in vitro insulin sensitivity. Cell 171, 372-384 e312. https://doi.org/10.1016/j.cell.2017.08.035 (2017).

28. Zhang, B., Yang, Y., Xiang, L., Zhao, Z. \& Ye, R. Adipose-derived exosomes: a novel adipokine in obesity-associated diabetes. J. Cell. Physiol. https://doi.org/10.1002/jcp.28354 (2019).

29. Thomou, T. et al. Adipose-derived circulating miRNAs regulate gene expression in other tissues. Nature 542, 450-455. https://doi. org/10.1038/nature21365 (2017).

30. Goody, D. \& Pfeifer, A. BAT exosomes: metabolic crosstalk with other organs and biomarkers for BAT activity. Handb. Exp. Pharmacol. 114, 1-10. https://doi.org/10.1007/164_2018_114 (2018).

31. McCormick, R. I. et al. miR-210 is a target of hypoxia-inducible factors 1 and 2 in renal cancer, regulates ISCU and correlates with good prognosis. Br. J. Cancer 108, 1133-1142. https://doi.org/10.1038/bjc.2013.56 (2013).

32. Grosso, S. et al. MiR-210 promotes a hypoxic phenotype and increases radioresistance in human lung cancer cell lines. Cell Death Dis. 4, e544. https://doi.org/10.1038/cddis.2013.71 (2013).

33. Hondares, E. et al. Thermogenic activation induces FGF21 expression and release in brown adipose tissue. J. Biol. Chem. 286, 12983-12990. https://doi.org/10.1074/jbc.M110.215889 (2011).

34. Suh, J. M. et al. Endocrinization of FGF1 produces a neomorphic and potent insulin sensitizer. Nature 513, 436. https://doi. org/10.1038/nature13540 (2014).

35. Fisher, F. M. et al. FGF21 regulates PGC-1 and browning of white adipose tissues in adaptive thermogenesis. Genes Dev. 26, 271-281. https://doi.org/10.1101/gad.177857.111 (2012).

36. Lewis, J. E. et al. Antibody-mediated targeting of the FGFR1c isoform increases glucose uptake in white and brown adipose tissue in male mice. Endocrinology 591, 1-10. https://doi.org/10.1210/en.2017-00591 (2017).

37. Sun, H. D. et al. Monoclonal antibody antagonists of hypothalamic FGFR1 cause potent but reversible hypophagia and weight loss in rodents and monkeys. AJP Endocrinol. Metab. 292, 964-976. https://doi.org/10.1152/ajpendo.00089 (2006).

38. Chen, Y. et al. Exosomal microRNA miR-92a concentration in serum reflects human brown fat activity. Nat. Commun. 7, 11420. https://doi.org/10.1038/ncomms11420 (2016).

\section{Acknowledgements}

This work was supported by the National Natural Science Foundation of China (31571231); Qinghai International Collaboration Program (2015-HZ-807); Guiding Program of Qinghai Provincial Health Commission (2019-wjzdx-04).

\section{Author contributions}

B.Z. and G.R. conceived and designed the experiments, B.Z. wrote the manuscript, Z.Y. analysed, interpreted results. Z.Y., S.K., and Y.R. performed animal experiments and measurement of parameters. S.K. performed mitochondrial respiration measurement with O2K. Q.G., Y.Y., L.Y., and W.S. run PET-CT scan. G.R. contributed to proof-reading and correction of manuscript.

\section{Competing interests}

The authors declare no competing interests.

\section{Additional information}

Supplementary information is available for this paper at https://doi.org/10.1038/s41598-020-71345-8.

Correspondence and requests for materials should be addressed to Z.B. or R.G.

Reprints and permissions information is available at www.nature.com/reprints.

Publisher's note Springer Nature remains neutral with regard to jurisdictional claims in published maps and institutional affiliations.

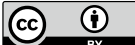

Open Access This article is licensed under a Creative Commons Attribution 4.0 International License, which permits use, sharing, adaptation, distribution and reproduction in any medium or format, as long as you give appropriate credit to the original author(s) and the source, provide a link to the Creative Commons licence, and indicate if changes were made. The images or other third party material in this article are included in the article's Creative Commons licence, unless indicated otherwise in a credit line to the material. If material is not included in the article's Creative Commons licence and your intended use is not permitted by statutory regulation or exceeds the permitted use, you will need to obtain permission directly from the copyright holder. To view a copy of this licence, visit http://creativecommons.org/licenses/by/4.0/.

(c) The Author(s) 2020 